\title{
ESTUDIO TIPOLÓGICO ARQUITECTÓNICO DE LAS BODEGAS EN EL VALLE DE LOCUMBA
}

\section{ARCHITECTURAL TIPOLOGICAL STUDY OFTHE WINERIES IN LOCUMBA VALLEY}

\author{
Gustavo Becerra Moscoso , Jaime Pinto Delgado , Elmer Rivera Mansilla ${ }^{2}$, Ivan Chura Chino ${ }^{3}$, Jersson Castilla Veliz ${ }^{3}$
}

\section{RESUMEN}

El presente estudio se desarrolló en el Valle de Locumba y anexo del valle de Cinto, perteneciente a la hidrografia del rio Locumba hacia el Océano Pacifico, Departamento de Tacna. El análisis de tipologías arquitectónicas de las bodegas en dicho valle han permitido visualizar a nivel del conjunto de bodegas que estuvieron en su apogeo de producción de vino y luego pisco, entre los siglos XVIII y XIX, $y$ que se integraron mediante un sistema espacial desconcentrado por cada valle, $y$ donde se encontraron méritos arquitectónicos que expresaron tipologias generales en lo espacial, funcional y formal, y que asimismo esto posibilitará posteriores estudios de detalle a nivel del estudio tipológico, que permitan ejecutar proyectos para su recuperación y puesta en valor para convertirlos en testimonios históricos, yarquitectónicos y su consiguiente valor como recurso y destinos turísticos.

Palabras clave: tipologias arquitectónicas, bodegas de vino, estudio tipológico.

\section{ABSTRACT}

This study was developed in Locumba Valley and annex of Cinto valley that belong to the Locumba River's hydrography toward the Pacific Ocean, in Tacna Department. The analysis of architectural typologies of wineries in this valley has enabled display them to the set level that they were at their peak production of wine and pisco, between the eighteenth and nineteenth centuries. The wineries were joined by a devolved spatial system for each valley, and there architectural merits that showed general typologies expressed in the spatial, functional and formal were found. Also this will enable further detailed studies to a typological study level that will allow them to implement projects for their recovery and enhancement to become wineries into historical and architectural evidence and they obtain their consequent value as resource and tourist destinations.

Keywords: architectural typologies, wineries, typological study.

\section{INTRODUCCIÓN}

El estudio se desarrolló en el valle de Locumba y anexo de Cinto, perteneciente a la hidrografía del río Locumba, hacia el Océano Pacífico en el Departamento de Tacna, donde se analizó tipologías arquitectónicas en términos de emplazamiento de las bodegas. El estudio viene a significar un posicionamiento físico espacial que identifique las resultantes tipológicas arquitectónicas que se tuvieron en los emplazamientos de las bodegas a lo largo del valle de Locumba y Cinto, este estudio toma relativa importancia dado que el Perú fue el país que tuvo los primeros viñedos y bodegas de América, siendo en este contexto, las bodegas los recintos tradicionales para la elaboración del vino y pisco, muchas de las cuales a la fecha cumplen dicha función en los valles de Lima, Ica, Arequipa, Moquegua y Tacna, y en varios casos son destinos turísticos.

En la región Tacna, los valles de Locumba y Caplina, desde los siglos XVI al XX, según cita Lévano en su libro "CINTO, Valle Maravilloso" (2006) fueron los primeros productores de vinos y aguardientes en la zona sur del Perú, y de buena acogida en mercados europeos, y han tenido un desarrollo expectante en el tema, pero por desbalances en los ecosistemas dichos cultivos caducaron; ante esto la experiencia del Valle de Moquegua es quizá la más cercana en el tiempo y en lo espacial al tema, dado que la economía colonial de Moquegua se basó en la agroindustria del vino y del

\footnotetext{
${ }^{1}$ Arquitecto. Facultad de Ingeniería Civil, Arquitectura y Geotecnia de la Universidad Nacional Jorge Basadre Grohmann. Correoelectrónico:gubemo@yahoo.com;ipinto112000@yahoo.es

${ }^{2}$ Doctor en Ciencias Sociales. Facultad de Educación, Comunicacióny Humanidades de la Universidad Nacional Jorge Basadre Grohmann.

${ }^{3}$ Estudiante. Facultad de Ingeniería Civil, Arquitecturay Geotecnia de la Universidad Nacional Jorge Basadre Grohmann.
} 
pisco desde el siglo XVI, y donde según el estudio de Prudence M. Rice, se identificaron 130 sitios de bodegas con instalaciones para prensar, fermentar y almacenar el vino, destilándolo en aguardiente, y la fabricación de vasijas de barro utilizadas para el almacenamiento (tinajas) y el transporte (botijas).

El estudio pretende identificar la problemática de la situación actual de las bodegas del valle de Locumba, para su registro y catalogación, su tipificación arquitectónica, su valoración cultural como patrimonio de la nación, a fin de generar estudios, planes y otros proyectos a futuro, y generar un desarrollo turístico a partir de los atractivos y méritos arquitectónicos de las casas bodega, además recuperar la identidad cultural-social y preservarlas en el tiempo.

\section{Objetivo General}

Identificar y determinar las tipologías arquitectónicas de las bodegas del valle de Locumba a partir de su inventario, registro y catalogación funcional, espacial y formal.

Las resultantes espaciales que para su desagregado tipológico, tenían que partir de un contexto de definición según su morfología, se asocian al estudio que hace sobre imagen, en lo referente a las tipologías espacial y formal a partir de pequeños barrios o distritos conformados por la adición de unidades dentro de un conjunto y que se emplazan en un ensamble morfológico que, para la época, guardaba armoniosa relación de diálogo entre lo edificado y el entorno natural circundante, y que a la fecha ha sido modificado por las nuevas actividades agropecuarias.

\section{ANTECEDENTES}

Los antecedentes, se refieren a las experiencias afines que se tienen en relación al estudio y sus temas complementarios, tanto a nivel de las bodegas en si y sus emplazamientos, como a referencias bibliográficas sobre análisis tipológico; esto se ha desagregado en lo siguiente:

\section{Proceso de pisco y vino en bodegas del Perú}

A nivel nacional, y sobre todo en la zona centro y sur de la costa, desde hace dos siglos aproximadamente, se explotaron las áreas agrícolas con el cultivo de la uva y la aceituna, traídos por los españoles, y dada la bondad agrológica de los valles, se generó un valor agregado que a la fecha no alcanza su verdadera explotación y valor.

En este contexto, los recintos tradicionales para la elaboración del vino y pisco eran las bodegas, muchas de las cuales a la fecha cumplen dicha función en los valles de Lima, Ica, Arequipa, Moquegua y Tacna, y en varios casos, estas son destinos turísticos.

\section{Bodegas en Tacna y Moquegua}

En la región Tacna, los valles de Locumba y Caplina, desde los siglos XIX y XX, tuvieron un desarrollo expectante en el tema, pero no hay logros efectivos, tanto a nivel de planeamiento, inventario y de recuperación de zonas tradicionales como de la producción en escala y comercialización a otros mercados, siendo las zonas de Magollo y del Valle Viejo, donde se encuentran experiencias relacionadas al tema.

A mediados del siglo XVI, algunos españoles buscaron en la región nuevas tierras para sembrar uvas, trigo, caña de azúcar y otros cultivos, y así encontraron fértiles tierras en Moquegua, siendo la vid uno de los cultivos de mayor éxito, por su clima era un lugar ecológico muy adecuado para la viticultura hecho que fue acogido por la población europea. Fueron los españoles por su tradición en el cultivo y consumo de vinos, los que mejor explotaron dichos cultivos y la agroindustria de la vid en piscos y vinos.

El cultivo de la vid en Moquegua data desde los primeros años del Virreinato. Los españoles trajeron pequeñas cepas produciéndose en el valle vinos y aguardiente (pisco) que deleitaron a la corte de la Ciudad de los Reyes y los Virreinatos vecinos al sur

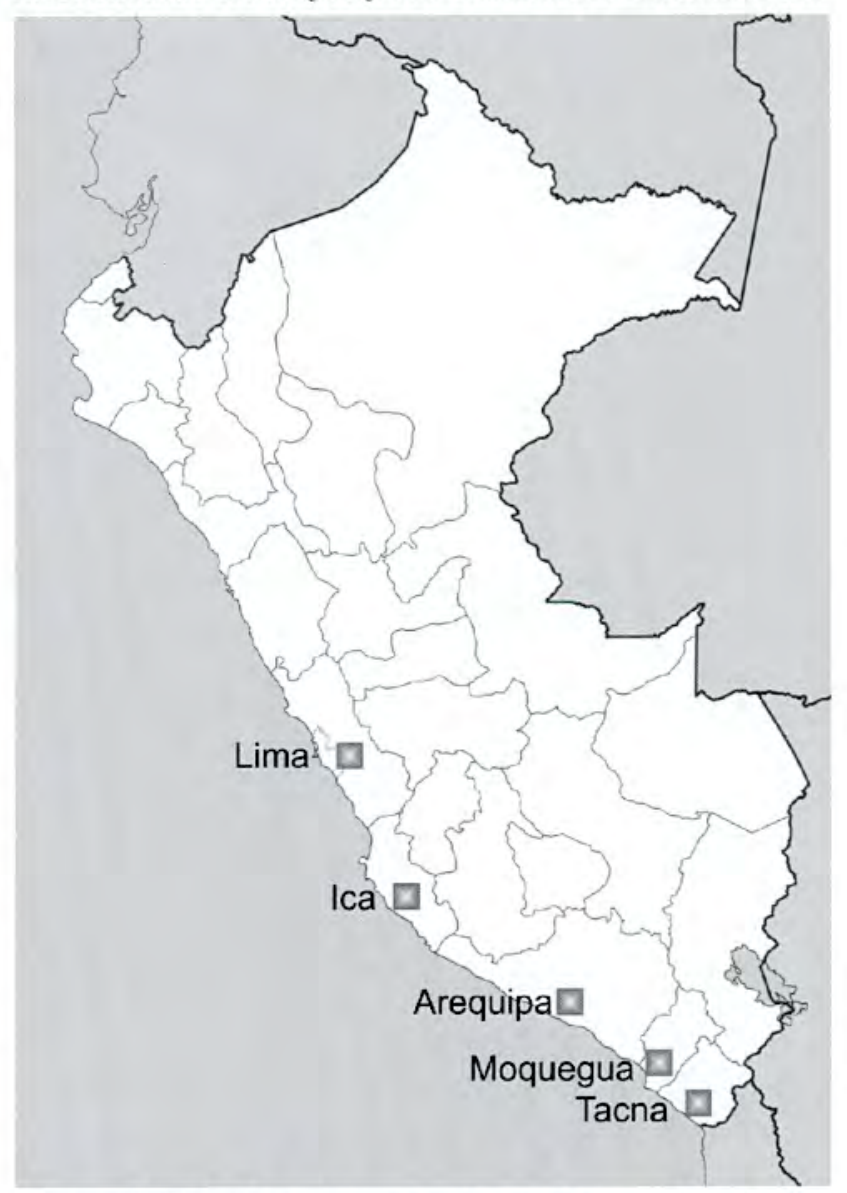

Figura $N^{\circ} 01$. Emplazamiento centros vitivinícolas $\mathrm{y}$ bodegas en la faja costera peruana 

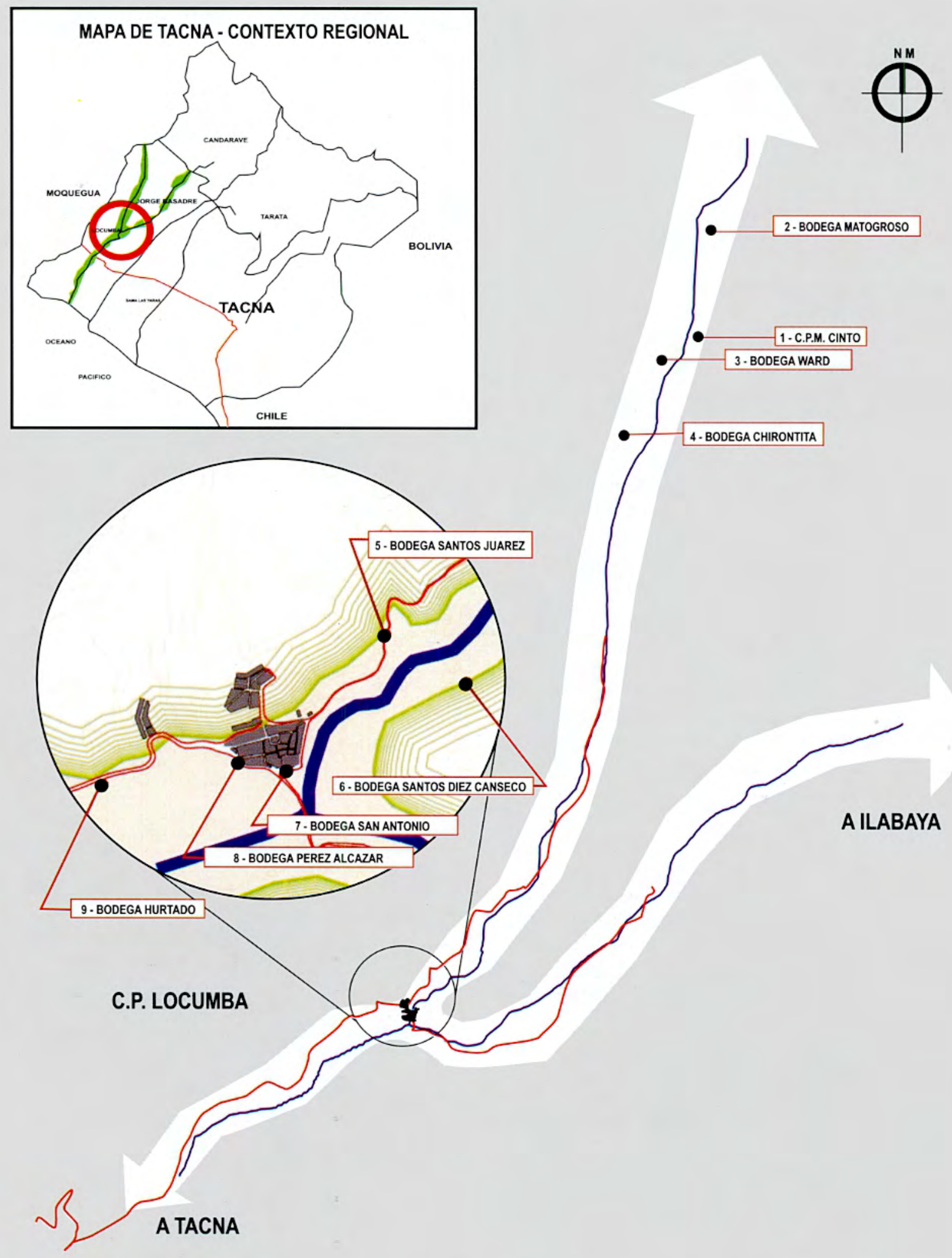

SISTEMA ESPACIAL DEL EMPLAZAMIENTO TERRITORIAL DE LAS BODEGAS

Figura $N^{\circ} 02$. Emplazamiento de bodegas en el sub sistema del valle Locumba 
En turismo, las bodegas se convierten en importantes destinos turísticos. En Moquegua la ruta del pisco nos permite introducirnos en un mundo centrado en la producción de vino y pisco, que se mantiene en bodegas, viñedos y personajes de antigua y señorial tradición que persiste hasta nuestros días.

\section{Emplazamiento vitivinícola en el Perú}

El emplazamiento espacial de las bodegas en la región de la costa y valles costeños del Perú, se dio con significación vitivinícola desde el siglo XVI y comprendió un sistema lineal respecto a la costa peruana siendo los valles más significativos: valle de Mala y Cañete en Lima / valles de Chincha y de Ica, en Ica / valles de Yauca, Majes y Vítor en Arequipa / valle de Moquegua / valle de Locumba en Tacna.

\section{ANÁLISIS ESPACIAL}

La zona de estudio se ubica en la provincia Jorge Basadre, a $80 \mathrm{~km}$ aproximadamente de la ciudad de Tacna, y se accede desde la carretera Panamericana Sur, encontrándose el Centro Poblado a 12,9 km de dicha intersección vial, a la altura del puente Locumba. La geo-referencia es la siguiente:

Latitud Sur: $17^{\circ} 36^{\prime} 35^{\prime \prime}$

Longitud Oeste: $70^{\circ} 45^{\prime} 39^{\prime \prime}$

En el estado situacional de las bodegas en general, se ha observado el deterioro generalizado que hay en las ocho bodegas, y en algunos casos el colapso de sus estructuras y zonas que en algunas veces no permite realizar la conjetura tipológica que le corresponda a cada bodega; dicho estado permite mostrar patologías o deterioros que sumados al impacto de la reforma agraria o el resto de agua y la contaminación generada por la empresa minera SPC de Toquepala, ha dado como resultante una crisis arquitectónica en la arquitectura de valor de las bodegas; este estado negativo y que linda con la

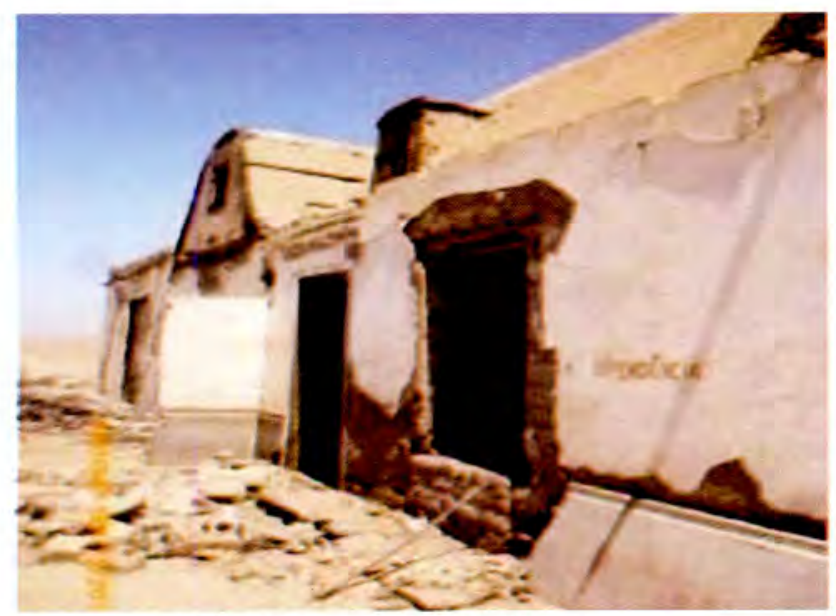

Figura $\mathrm{N}^{\circ} 03$. Colapso de bodegas pérdida definitiva del valor patrimonial arquitectónico y de mérito histórico de los vestigios de las bodegas en los valles indicados, se resumen genéricamente en lo siguiente estados de daños:

- Colapso de muros y techos de la zona residencial y la zona de bodega, producto de sismos, presiones de aire y erosión provocada por los mismos.

- Pérdida del mortero y parantes de columnas de madera, por erosión y robos desus partes.

- Fisuras, agrietamientos, grietas en muros y pisos, con pérdida del nivel vertical $u$ horizontal.

- Pérdida general de los $2 / 3$ de altura en muros de adobe, así como un casi $50 \%$ de la base de piedra de cantería.

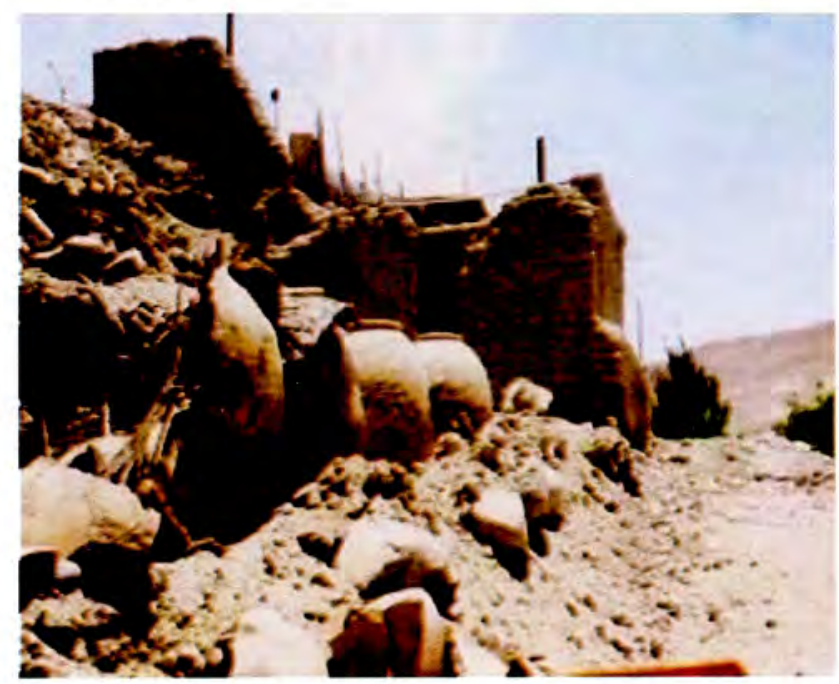

Figura $\mathrm{N}^{\circ} 04$. Pérdida de estructura

- Ciclos rasos y cobcrturas con pérdida de entramados de carrizos y barro, y presencia orgánica patógena en viguerías y pies derechos y columnas de madera.

- Pisos desnivelados, con asentamientos diferencial bien marcado, respecto al nivel original.

- Detcrioro y pérdida de un $50 \%$ o mas de la vasijas o tinajones de las bodegas, estando muchas de ellas

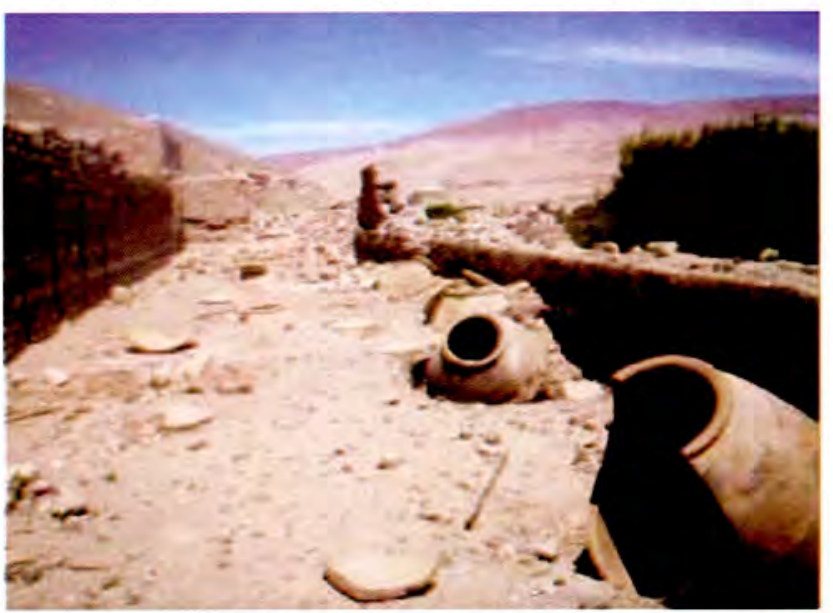

Figura $\mathrm{N}^{\circ} 05$. Deterioro de vasijas y tinajones 


\section{La Vida y la Histaria}

Becerra, G. et al. Estudio Tipológico Arquitectónico de las Bodegas en el Valle de Locumba

fuera de su lugar original.

- Presencia de humedad y desechos sobre elementos de valor patrimonial, como adobes, tinajones

El estudio pretende identificar la problemática de la situación actual de las Bodega del Valle de Locumba, para:

- Su registro y catalogación

- Su tipificación arquitectónica

- Su valoración cultural como patrimonio de la nación

- Para generar estudios, planes y otros proyectos a futuro

- Para generar un desarrollo turístico a partir de los atractivos y méritos arquitectónicos de las casas bodegas

- Para recuperar la identidad cultural-social y preservarlas en el tiempo

Para implementar este análisis espacial los procedimientos y metodología empleados tuvieron como base la definición de una Investigación Aplicada y Descriptiva, donde luego de la observación de los contextos temáticos definimos las fases de los niveles de estudio: Visitas, Muestras, Sistematización de información, Redefinición de las Variables y verificación de hipótesis y se estudió lo comparativo y correlacionado de las variables de estudio.

Las técnicas e instrumentos de recolección de datos fueron: Observación directa en campo, Entrevistas con autoridades y pobladores, cuestionarios y encuestas, fichas de campo y registros fotográficos, bibliografías relacionadas, levantamiento de planos.

Como método y técnica de procesamiento y de análisis se utilizó la técnica del análisis compositivo arquitectónico para definir la morfología y estilística arquitectónica de emplazamiento de bodegas a través de las variables de estudio, y luego un análisis comparativo, expresando ello en cuadros y gráficos estadísticos de los registros de campo, como de los procesos resultantes del análisis de las variables.

La contrastación y verificación de hipótesis se ejecutó bajo las bases de las variables formales, espaciales y funcionales y sus respectivas dimensiones $\mathrm{e}$ indicadores.

\section{RESULTADOS TIPOLÓGICOS}

Desde su emplazamiento pre hispánico, Locumba ha tenido un crecimiento espontáneo tanto a nivel de sus centros poblados como de sus áreas naturales y las agrícolas, que a su vez tenían una lógica territorial de emplazamientos, buscando que los asentamientos o conjuntos arquitectónicos como las bodegas, estén diferenciados del área agrícola o de zonas naturales de riesgo.

Ante ello, la resultante de estudio tanto a nivel de emplazamiento de los centros poblados o conjuntos de hechos arquitectónicos y el sistema de las bodegas es un todo armónico.

\section{Emplazamiento: Organización espacial del sistema de Bodegas}

El presente estudio de las tipologías de las bodegas en el valle de Locumba, y adicionalmente luego de las visitas de campo, en el valle de Cinto, nos permite aseverar que es importante el reconocer su aporte al legado arquitectónico y patrimonial de Tacna. Esto implica instalar el tema tipológico dentro de un contexto espacial y territorial de la costa peruana y el nivel regional y local, y tomamos como base el estudio que hace Spreigeren(1973:81-83) en el capítulo Análisis Visual de su libro "Compendio de Arquitectura Urbana", donde plantea para el caso de visualizar la forma urbana, el análisis del grado de emplazamientos de los componentes a inter relacionar, y en el caso se tiene que localizar los restos de las bodegas.

En Locumba, este modelo de emplazamiento se daba con las bodegas adyacentes a una vía (trocha) y con dominio visual y de acceso a la zona agrícola de viñedos que eran regados con agua del río, y adyacente se ubicaba la vivienda del propietario de la bodega y en algunos casos la residencia para los asalariados.

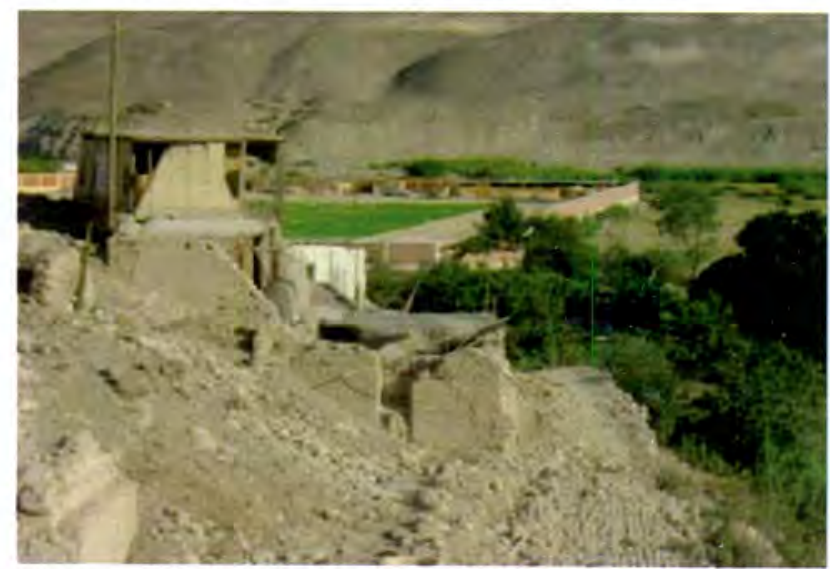

Figura $\mathrm{N}^{\circ} 06$. Bodega adyacente a una vía

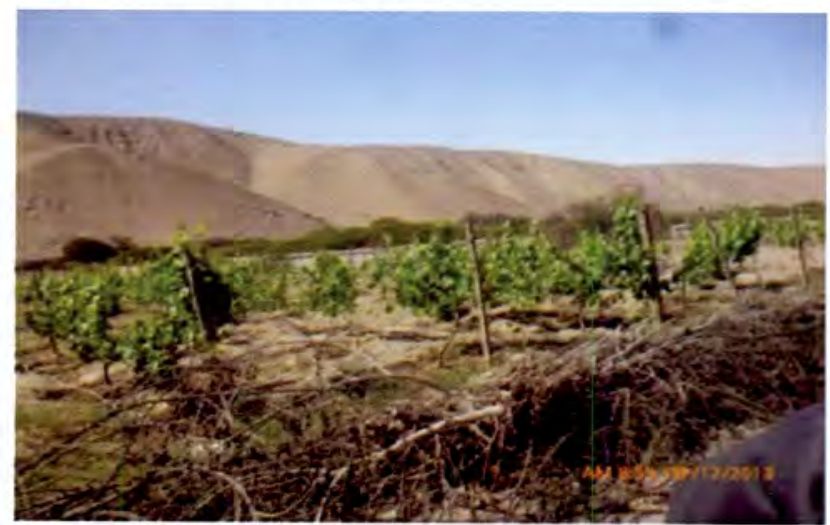

Figura $\mathrm{N}^{\circ} 07$. Vista de zona de viñedos 


\section{Identificación del sistema de bodegas en el ecosistema Locumba - Cinto}

En el estudio se ha determinado diferentes indicadores, tanto para la variable independiente (tipologías), como para la variable dependiente (bodegas), y que permiten desagregar dicho estudio en las variables tipológicas: Funcionales (FUN), Formales (FOR) y Espaciales (ESP), que a su vez desagregan las dimensiones e indicadores anotadas en la matriz de consistencia.

Se entiende que la tipología arquitectónica es producto de un previo análisis y percepción del espacio, como lo cita Acuña (2000) en su libro "Fundamentos de Planeamiento Urbano Aspectos Técnicos" y nos permite asociar, agrupar, homogenizar criterios en la observación y poder sistematizar dichas tipologías, y deducir órdenes o parámetros análogos de los hechos arquitectónicos, desde lo contextual, hasta el hecho arquitectónico integrado expresado en conjunto o en unidades arquitectónicas.

Los modelos de emplazamiento ruralarquitectónico de las bodegas en los valles de Locumba y Cinto, obedecen a que los ducños de haciendas optaron por emplazarlas adyacentes a sus áreas agrícolas de viñedos, procurando no ocupar áreas con aptitud para el cultivo.

En este aspecto tenemos los siguientes tipos genéricos de emplazamiento con las siguientes zonas y/o hechos arquitectónicos: La Casa. La Bodega, El Patio y/o Corral, Los Viñedos y el Acceso Vía. (Ver Figuras $\mathrm{N}^{\circ} 08$ y N $\mathrm{N}^{\circ} 09$ )

Cada una de estas zonas o sectores tenían a su vez componentes interiores, que serán materia de un futuro cstudio más espccífico.

Los tipos de emplazamiento que expresan una modelación síntesis son:

Emplazamiento Tipo 1: Ubica a la casa separada del resto de zonas, esta tipología se forma por una vía trocha; adyacente a ésta y en frente opuesto a la casa, se ubica la bodega en sí, que perimetra el patio en parte, $y$ luego se ubican los viñedos autiguos $y$ actualmente las zonas agrícolas.

Emplazamiento Tipo 2: Ubica a todo el conjunto adyacente a la vía- trocha, y con la ubicación referente a la tipología anterior de la casa adjunta a la vía; luego ubica la bodega, el patio y en forma circundante los viñedos.

Emplazamiento Tipo 3: Tipología similar a la anterior, con la diferencia que cl entorno al cortijo está con los viñedos, hacia la parte posterior y con zonas criazas en los lados laterales del conjunto.

Emplazamiento Tipo 4: Esta tipología se ubica en una zona topográfica elevada (cerro), estando la casa, la bodega y el patio en la zona eriaza de la misma, y en el nivel más bajo el área agrícola de los viñedos.
Estos emplazamientos tipológicos. que se dieron en los dos siglos pasados, son de índole general y de relativo estándar, y que en todos los casos responden por respeto al área agrícola de los viñedos.

En cuanto a las evidencias encontradas, en las visitas de estudio se ve que en su mayor parte la infraestructura descrita está en ruinas.

Ante ello desagregaremos las siguientes tipologías:

\section{a) Tipología Formal}

Es quizá la tipología que más evidencia las características peculiares del hecho arquitectónico, por su emplazamiento en el contexto, o como grado de composición en unidad arquitectónica. o como conjunto arquitectónico adosado en unidades, y que a modo de "barrios" o "distritos" como cita Lynch(1959) en su libro "La Imagen de la Ciudad", expresan conjuntos con unidades del tipo "Cuarto" que nos cita Montenegro(2010) en su Libro "Estudio Tipológico de la Vivienda Tacneña".

Tabla N01: Tipologías Formales según su Emplazamiento Físico Espacial (T-For)

TIPOLOGIA

EMPLAZAMIENTO

VALLE ALTO VALLE BA.JO

Tipologia en Conjunto Hacienda Wari

Arquitectónico Bodega Chironta

(T1-FOR-BCA)

\begin{tabular}{|c|c|c|}
\hline \multirow{6}{*}{$\begin{array}{l}\text { Tipologia en Unidad } \\
\text { Arquitectónica } \\
\text { (T2-FOR-BUA): }\end{array}$} & Bodega Matto & Bodega San Autonio \\
\hline & Grosso & Bodega Diez Canseco \\
\hline & & Bodega Santo: Juares \\
\hline & & Bodega PerezAlcazar \\
\hline & & Bodega Hurtado \\
\hline & & Bodega Valdivia \\
\hline
\end{tabular}

\section{b) Tipología Espacial}

Se ha determinado la tipología espacial segúm su localización en el sistema de los valles de Cinto y Locumba, el grado de emplazamiento y por el tipo de geo forma, que el Arq. Percy Acuña Vigil la expresa en el capítulo Análisis y Percepción del Espacio, del Libro: Fundamentos de Planeamiento Urbano Aspectos Técnicos, en que se emplaza cada bodega. y que se regían por las condicionantes de: Topografía, accesibilidad, aptitud de suelo, y cercanía a recurso hídrico: ante ello se han determinado tres tipologías de bodegas:

\section{c) Tipología Funcional}

En los valles de estudio se distinguen, luego de las indagaciones de fuentes del lugar, y de la observación in situ, que básicamente se definen las actividades de habitar, de producción, y de comercialización.

La zonificación típica en las bodegas, que se visualizan en las Figuras $\mathrm{N}^{\circ} 08$ y $\mathrm{N}^{\circ} 09$, se da con las 


\section{La Vida y la Fistaria}

Becerra, G. et al. Estudio Tipológico Arquitectónico de las Bodegas en el Valle de Locumba

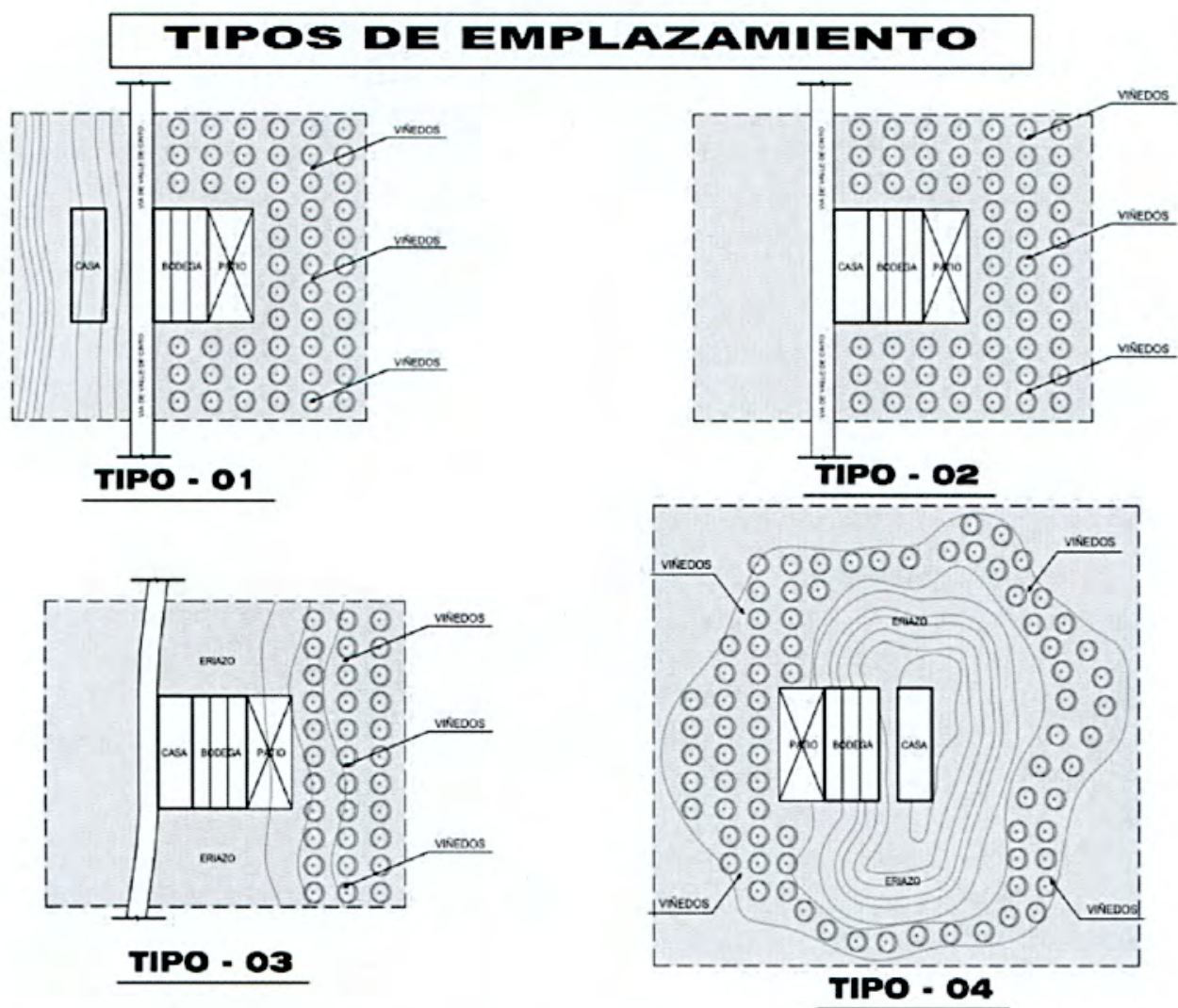

Figura $\mathrm{N}^{\circ} 08$. Emplazamientos típicos de bodegas. Tipos de emplazamiento
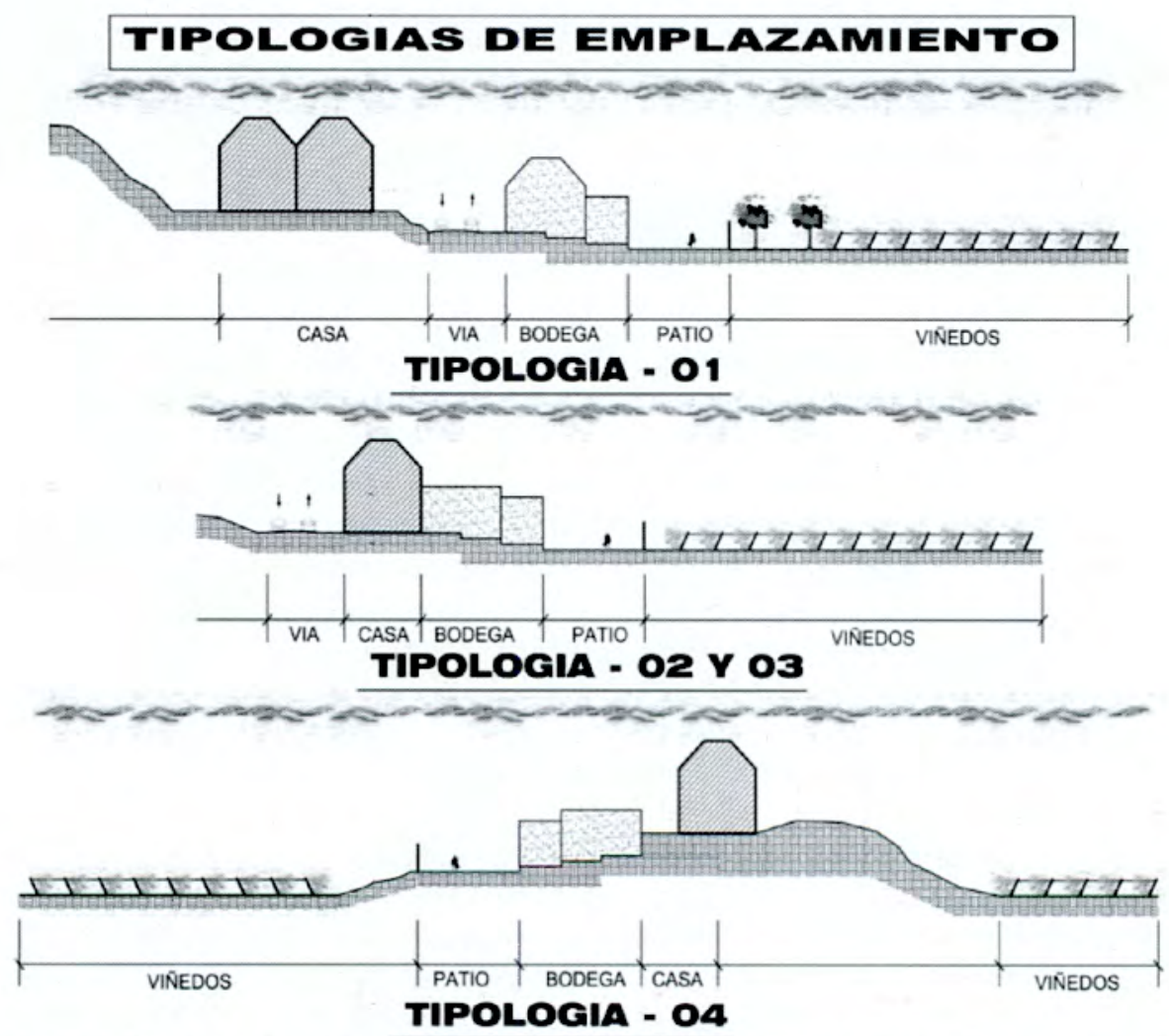

Figura $N^{\circ} 09$. Emplazamientos típicos de bodegas. Tipología de emplazamiento 

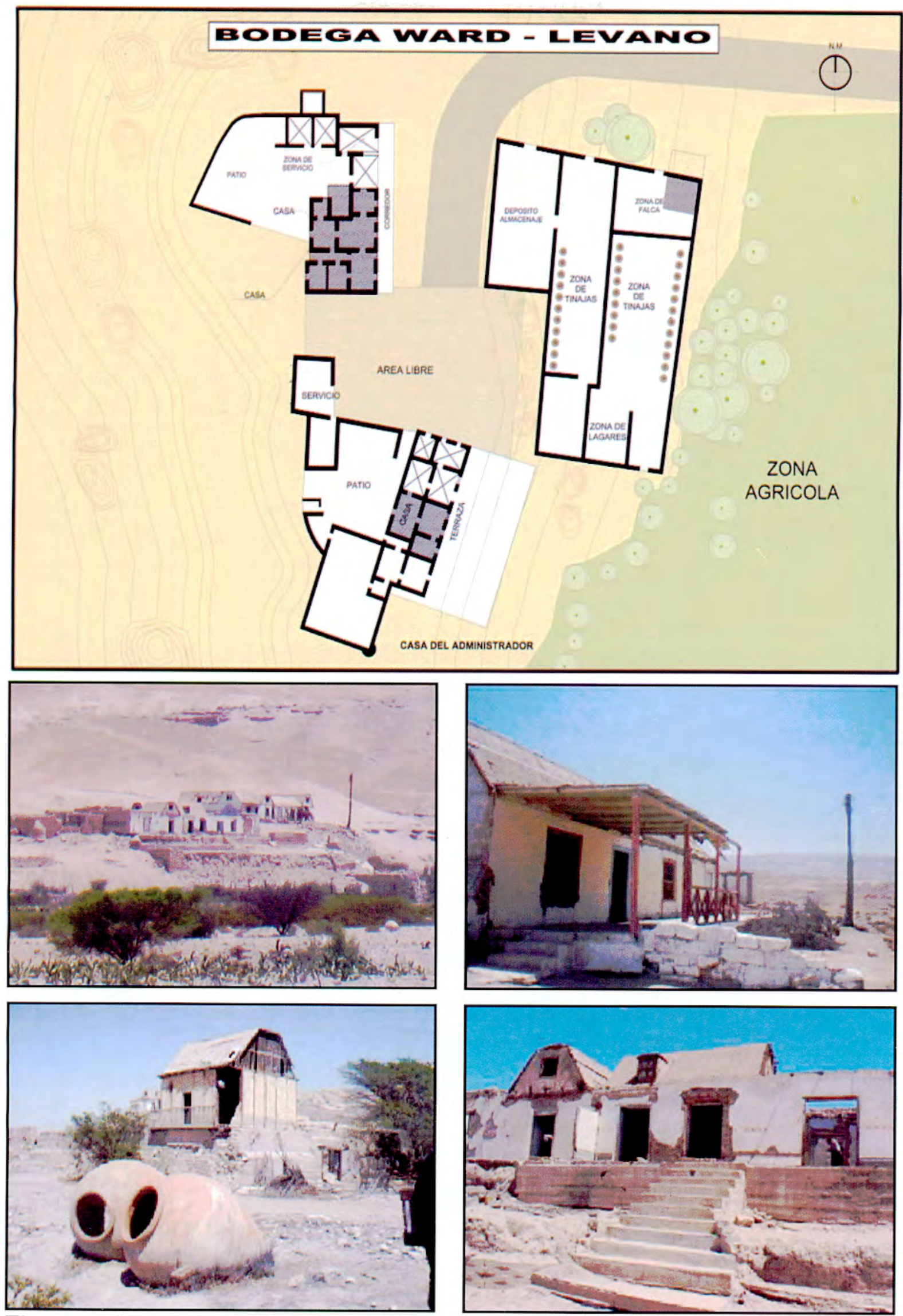

Figura 10. Vistas de emplazamiento conjunto arquitectónico. Bodega Ward-Levano 
Tabla N02: Tipologías por localización (T1-Esp)

\begin{tabular}{|c|c|c|}
\hline \multirow[t]{2}{*}{ TIPOLOGÍA } & \multicolumn{2}{|c|}{ EMPLAZAMIENTO } \\
\hline & VALLE ALTO & VALLE BAJO \\
\hline \multicolumn{3}{|c|}{ Bodegas Valle Alto Bodega Mato Grosso } \\
\hline \multirow[t]{3}{*}{ T1-ESP-BVA } & Hacienda Ward & \\
\hline & Bodega Chironta & \\
\hline & Bodega Diez Canseco & \\
\hline \multirow{4}{*}{\multicolumn{2}{|c|}{$\begin{array}{c}\text { Bodegas Valle Bajo } \\
\text { T1-ESP-BVB }\end{array}$}} & Bodega San Antonio \\
\hline & & Bodega PćrezAlcázar \\
\hline & & Bodega Hurtado \\
\hline & & Bodega Valdivia \\
\hline
\end{tabular}

Tabla $\mathrm{N}^{\circ} 03$ : Tipología según ámbito de emplazamiento (T2-Esp)

\begin{tabular}{|c|c|}
\hline ÁMBITO & BODEGAS \\
\hline $\begin{array}{l}\text { En ámbito Rural } \\
\text { (T2-ESP-BR) }\end{array}$ & $\begin{array}{l}\text { Bodega Matto Grosso } \\
\text { Hacienda Ward } \\
\text { Bodega Chironta }\end{array}$ \\
\hline $\begin{array}{l}\text { En ámbito semi Urbano } \\
\text { (T2-ESP-BSU) }\end{array}$ & $\begin{array}{l}\text { Bodega Diez Canseco } \\
\text { Bodega Santos Juares } \\
\text { Bodega Hurtado } \\
\text { Bodega Valdivia }\end{array}$ \\
\hline $\begin{array}{l}\text { En ámbito Urbano } \\
\text { (T2-ESP-BU) }\end{array}$ & $\begin{array}{l}\text { Bodega San Antonio } \\
\text { Bodega PerezAlcazar }\end{array}$ \\
\hline
\end{tabular}

Tabla Nº4: Tipologías según su emplazamiento de Geo forma (T3-Esp)

\begin{tabular}{cl}
\hline \multicolumn{1}{c}{ GEOFORMA } & \multicolumn{1}{c}{ BODEGAS } \\
\hline Planicie (T3-ESP-BGP) & Bodega Chironta \\
& Bodega Santos Juares \\
& Bodega San Antonio \\
& Bodega Hurtado \\
& Bodega Valdivia \\
& Bodega Matto Grosso \\
Terraza (T3-ESP-BGT) & Hacienda Ward \\
& Bodega Diez Canseco \\
& Bodega PérezAlcázar \\
\hline
\end{tabular}

evidencias funcionales que se pudieron encontrar en vestigios y en alcances que dieron los pobladores. Estas zonas dan base a la tipología funcional con los usos de: vivienda, fabricación mas zona de Tornillos(vino y aguardientes), servicios, lagares (depósitos), hornos y el complemento agrológico de las zonas de cultivo, descritas en la siguiente figura.

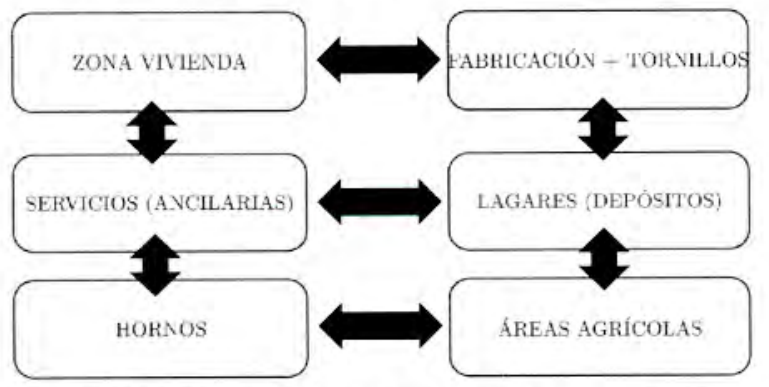

Figura $\mathrm{N}^{\circ} 11$. Zonificación funcional típica en bodegas
Tabla $N^{\circ} 05$ : Tipología Funcional (Fun)

\begin{tabular}{|c|c|c|}
\hline BODEGAS & TIPOLOGİA & $\begin{array}{c}\text { ACTIVIDADES / } \\
\text { FUNCIONES }\end{array}$ \\
\hline $\begin{array}{l}\text { Bodega Mato } \\
\text { Grosso }\end{array}$ & $\begin{array}{l}\text { Casa Bodega } \\
\text { Multifuncional } \\
\text { T-FUN-CBM }\end{array}$ & $\begin{array}{l}\text { Residencia unifamiliar, } \\
\text { producción agrícola y agro } \\
\text { industrial. }\end{array}$ \\
\hline Hacienda Ward $\frac{\mathrm{I}}{\mathrm{F}}$ & $\begin{array}{l}\text { I.a Casa Bodega } \\
\text { Polifuncional } \\
\text { T-FUN-CBP }\end{array}$ & $\begin{array}{l}\text { Residencia multifamiliar, } \\
\text { producción agrícola y agro } \\
\text { industrial, horneado de } \\
\text { tinajas. }\end{array}$ \\
\hline Bodega Chironta 1 & $\begin{array}{l}\text { La Casa Bodega } \\
\text { Polifuncional } \\
\text { T-FLN-CBP }\end{array}$ & $\begin{array}{l}\text { Residencia multifamiliar, } \\
\text { producción agrícola y agro } \\
\text { industrial, horneado de } \\
\text { tinajus. }\end{array}$ \\
\hline $\begin{array}{l}\text { Bodega Diez } \\
\text { Canseco }\end{array}$ & $\begin{array}{l}\text { Casa Bodega } \\
\text { Multifuncional } \\
\text { T-FUN-CBM }\end{array}$ & $\begin{array}{l}\text { Residencia unifamiliar, } \\
\text { producción agrícola y agro } \\
\text { industrial. }\end{array}$ \\
\hline $\begin{array}{c}\text { Bodega Santos } \\
\text { Juares }\end{array}$ & $\begin{array}{l}\text { Casa Bodega } \\
\text { Multifuncional } \\
\text { T-FUN-CBM }\end{array}$ & $\begin{array}{l}\text { Residencia unifamiliar, } \\
\text { producción agrícola y agro } \\
\text { industrial. }\end{array}$ \\
\hline $\begin{array}{c}\text { Bodega Sau } \\
\text { Antonio }\end{array}$ & $\begin{array}{l}\text { Casa Bodega } \\
\text { Multifuncional } \\
\text { T-FLN-CBM }\end{array}$ & $\begin{array}{l}\text { Residencia unifamiliar, } \\
\text { producción agrícola y agro } \\
\text { industrial. }\end{array}$ \\
\hline $\begin{array}{c}\text { Bodega } \\
\text { PerezAlcazar }\end{array}$ & $\begin{array}{l}\text { Casa Bodega } \\
\text { Wultifuncional } \\
\text { T-FUN-CBM }\end{array}$ & $\begin{array}{l}\text { Residencia unifamiliar, } \\
\text { producción agrícola y agro } \\
\text { industrial. }\end{array}$ \\
\hline Bodega Hurtado & $\begin{array}{l}\text { Casa Bodega } \\
\text { Multifuncional } \\
\text { T-FUN-CBM }\end{array}$ & $\begin{array}{l}\text { Residencia unifamiliar, } \\
\text { producción agrícola y agro } \\
\text { industrial. }\end{array}$ \\
\hline Bodega Valdivia & $\begin{array}{l}\text { Casa Bodega } \\
\text { Multifuncional } \\
\text { T-FUN-CBM }\end{array}$ & $\begin{array}{l}\text { Residencia unifamiliar, } \\
\text { producción agrícola y agro } \\
\text { industrial. }\end{array}$ \\
\hline
\end{tabular}

\section{DISCUSIÓN DE RESULTADOS}

Luego de los aspectos, temas desarrollados y encuadrados dentro una lógica de demostrar que significa el estudio del análisis tipológico, nos permite obtener y definir las tres Tipologías Base planteadas en el plan de trabajo del estudio, y que relacionadas entre si, nos dan una resultante físico espacial del hecho arquitectónico, ya sea como unidad o como conjunto arquitectónico

En cuanto a la adopción de tener una definición de tipologías enfocadas a lo contextual del estudio tipológico en relación a la ubicación y al emplazamiento de las bodegas en los valles de Locumba y Cinto, se fundamenta que las variables independientes, es decir las bodegas, no encuentran consolidadas en un espacio relativamente concentrado, sino mas bien la variable toma acepciones de emplazamiento territorial y tramos de intervalos espaciales en kilómetros, de ahí la definición del sistema Locumba-Cinto.

Este expuesto, tuvo un soporte referencial importante en la bibliografía tomada en cuanto a la diferente orientación del estudio tipológico, más igualmente válidas, entre la resultante tipológica que da el Arq. Luis Montenegro en su estudio sobre la vivienda tacneña, donde se concurre sobre los hechos arquitectónicos en si de la vivienda de esta ciudad, y define el tipo Cuarto como base de tal definición, y que 
también se asocia a lo que define Luis Cavagnaro Orellana en su Libro Tacna: Desarrollo Urbano y Arquitectónico (1536-1540), como tres Estilos: Cuarto, Casita y Casa según el número de cuartos y área ocupada. Ambos estudios dirigen sus definiciones al hecho arquitectónico en sí, y detallan su composición interna, que sumados a la data de fábrica, en los siglos XIX y XX, dan un prototipo tipológico sobre una arquitectura similar con el estudio tipológico de bodegas, en lo constructivo, en el momento histórico, grados de emplazamiento por unidad arquitectónica, y también como formas de respuesta ante los desastres naturales.

Para el caso, nuestro estudio tuvo una óptica. diferente en definir unas Tipologías Base en lo funcional, espacial y formal, y ahí instalar la variable dependiente de Bodegas, atendiendo otros aspectos de índole de emplazamiento territorial como contexto de su localización en el ecosistema Locumba - Cinto, y su grado de configuración como unidad o como conjunto de barrios o distritos, como define Kevin Lynch, a un conjunto de edificaciones dentro de un recinto.

Finalmente indicamos que la resultante tipológica nace de atributos del sistema de emplazamiento espacial de las nueve bodegas estudiadas, y la relación de configuración funcional, espacial y formal, y que responde a una aproximación de tipo contextual, para poder proseguir con otros estudios más específicos y de carácter interdisciplinario, de ser el caso.

\section{CONCLUSIONES}

El análisis ha determinado que es posible realizar el análisis tipológico arquitectónico en las nueve bodegas estudiadas, y poder identificar las tipologías en las mismas a nivel formal, espacial y funcional, y que posibilite su registro, inventario y futura puesta en valor.

Las resultantes espaciales, que para su desagregado tipológico, han partido de un contexto de definición según su morfología, tal como lo cita Lynch la Imagen de la ciudad, en lo referente a asociar la tipología espacial y formal a partir de pequeños barrios o distritos conformados por la adición de unidades dentro de un conjunto y como éstos se emplazan.

Se concluye que el patrimonio arquitectónico de la arquitectura de valor de las bodegas esta en riesgo de colapso por su abandono, falta de planes de preservación, y que sumados a los impactos históricos de la Reforma Agraria, el recorte de agua en la base productiva y económica en los fundos de los propietarios, nos da una resultante negativa y que linda con la pérdida definitiva del valor patrimonial de los vestigios de las bodegas en los valles indicados.
Referente a las tipologías formales se determina que existen dos tipologías formales que según su densidad, escala y grado de cohesión de edificaciones $\mathrm{y}$ que nos determinan los conjuntos o unidades arquitectónicas.

Se ha determinado tres tipologías espaciales existentes en el área de estudio. Estas tipologías están clasificadas según su localización en los valles de Locumba y Cinto, según el grado de emplazamiento y por la geo forma en que se emplaza cada bodega.

\section{RECOMENDACIONES}

Para poder definir, de una manera más precisa las tipologías arquitectónicas de las bodegas es necesario realizar estudios integrales y especializados en las diferentes disciplinas de arqueología, ingeniería, antropología, historia y agronomía, entre otras.

Las tipologías formales, por sus restringidas evidencias, y por sus características arquitectónicas; precisan de un análisis específico y detallado a nivel de características formales tales como: estilos, planos, volúmenes y detalles arquitectónicos.

Para la futura puesta en valor de las bodegas, se recomienda realizar estudios especializados a través de convenios con la Municipalidad Provincial de Jorge Basadre, el Gobierno Regional de Tacna, Ministerio de Cultura, las universidades y la empresa privada.

Gestionar y llegar a acuerdos de consenso con la empresa minera SPCC, para que participe en un plan integral de los valles, en cuanto a la recuperación de suelos, mejorar el balance hídrico, capacitaciones, y acciones de salvamento al patrimonio edificado de bodegas.

Dado el valor patrimonial e histórico de las edificaciones que antiguamente fueron bodegas, y en función de la seguridad de la población, se recomienda acciones de señalización protección y resguardo de elementos de valor que existen en dichas edificaciones, para tener evidencias reales ante un futuro proyecto integral.

\section{REFERENCIAS BIBLIOGRÁFICAS}

ACUNA, P (2000) Fundamentos de Planeamiento Urbano.

CAVAgnaro, L. (2010) Tacna: Desarrollo Urbano y Arquitectónico (1536-1880).

LÉVANO, L (2006) Cinto Valle Maravilloso.

LYNCI, K (1959) La Imagen de La Ciudad.

MONTENEGRO, L. (2010) Estudio Tipológico de La Vivienda Tacneña.

MORAES, R (2007) Trilogia do Patrimonio Histórico e Cultural Sul-Mato Grossense

RICE, P (1985) Bodegas de Moquegua.

SPREIGEREN (1973) Compendio de Arquitectura Urbana. 